\title{
Fragilidade e risco de queda em idosos que vivem no domicilio
}

Frailty and risk of falling in the older adult living at home

Fragilidad y riesgo de caída de ancianos que viven en su domicilio

Suelen Borelli Lima Giacomini' ${ }^{1}$ ittps://orcid.org/0000-0002-0611-8478

Jack Roberto Fhon ${ }^{1}$ i h htps://orcid.org/0000-0002-1880-4379

Rosalina Aparecida Partezani Rodrigues ${ }^{1}$ io https://orid.org/0000-0001-8916-1078

Como citar:

Giacomini SB, Fhon JR, Rodrigues RA.

Fragilidade e risco de queda em idosos que vivem no domicílio. Acta Paul Enferm. 2020;33:eAPE20190124.

DOI

http://dx.doi.org/10.37689/actaape/2020A00124

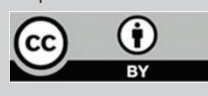

Descritores

Idoso; Acidentes por quedas; Idoso fragilizado; Envelhecimento; Saúde do idoso

Keywords Aged; Accidental falls; Frail elderly; Health of the elderly

Descriptores

Anciano; Accidentes por caídas; Anciano frágil, Envejecimiento; Salud del anciano

\section{Submetido} 21 de Maio de 2019

Aceito 21 de Outubro de 2019

Autor correspondente Rosalina Aparecida Partezani Rodrigues E-mail: rosalina@eerp.com.br

\section{Resumo}

Objetivo: Analisar a associação entre o risco de queda e a síndrome da fragilidade em idosos que vivem no domicílio.

Métodos: Estudo transversal com 261 idosos, de ambos os sexos, residentes em domicílios de um município paulista. A coleta ocorreu por meio dos instrumentos: Perfil Demográfico, Mini Exame do Estado Mental, Fall Risk Score, Edmonton Frail Scale, Indicador de Fragilidade de Tilburg e Indicador da fragilidade de Groningen. Para as análises bivariadas, utilizamos o teste de Qui-quadrado de Pearson e, para comparar as médias da escala numérica, o Teste não paramétrico de Mann-Whitney e a Regressão Logística Linear com p<0,05.

Resultados: Dos 261 idosos, a maioria era do sexo feminino, viúvas, com 1 a 4 anos de estudo e moravam com familiares. A prevalência do risco de queda foi de 51,7\%. Em todas as escalas utilizadas, houve associação entre fragilidade com 0 risco de queda $(p<0,001)$. Na análise de regressão logística linear, 0 idoso considerado frágil pela Escala de Tilburg apresentou 6,05 vezes mais chances de cair do que aquele não frágil. Na Escala de Groningen, as chances de o idoso frágil cair foram 5,55 vezes maiores e, na Escala de Edmonton, aqueles que apresentaram risco de queda obtiveram aumento de 1,53 na média do escore.

Conclusão: 0 risco de queda foi estimado com maior significância quando associado à fragilidade, nas três escalas utilizadas. Tais escalas são instrumentos de fácil acesso e aplicação por parte do enfermeiro e equipe multiprofissional e podem ser adotadas para favorecer um envelhecimento ativo.

\section{Abstract}

Objective: To analyze the association between risk of falling and frailty syndrome in the older adult living at home.

Methods: Cross-sectional study with 261 older adult men and women living in households in a city of São Paulo. The collection took place through the instruments: Demographic Profile, Mini Mental State Examination, Fall Risk Score, Edmonton Frail Scale, Tilburg Frailty Indicator and Groningen Frailty Indicator. For the bivariate analyzes, we used Pearson's chi-square test and, to compare the means of the numerical scale, the nonparametric Mann-Whitney test and the linear logistic regression with $p<0.05$.

Results: Of the 261 older adults, most were female, widows, with 1 to 4 years of education and living with family members. The prevalence of fall risk was $51.7 \%$. In all scales used, there was an association between frailty and risk of falling $(p<0.001)$. In the linear logistic regression analysis, the older adult considered frail by the Tilburg Scale were 6.05 times more likely to fall than the non-frail. On the Groningen Scale, the chances of the frail older adult falling were 5.55 times higher and, on the Edmonton Scale, those at risk of falling had a 1.53 average increase in the score. 
Conclusion: The risk of falling was most significantly estimated when associated with frailty, in the three scales used. Such scales are easily accessible and applicable instruments by nurses and multiprofessional staff and can be adopted to favor active aging.

\section{Resumen}

Objetivo: Analizar la relación entre el riesgo de caída y el síndrome de fragilidad de ancianos que viven en su domicilio.

Métodos: Estudio transversal con 261 ancianos, de ambos sexos, residentes en domicilios de un municipio de São Paulo. La recolección se realizó mediante Ios siguientes instrumentos: Perfil Demográfico, Mini Examen del Estado Mental, Fall Risk Score, Edmonton Frail Scale, Indicador de Fragilidad de Tilburg e Indicador de fragilidad de Groningen. Para el análisis bivariado, utilizamos la prueba $\bigotimes^{2}$ de Pearson, y para comparar los promedios de la escala numérica, la Prueba no paramétrica de Mann-Whitney y la Regresión Logística Lineal con $p<0,05$.

Resultados: De los 261 ancianos, la mayoría era de sexo femenino, viudas, entre 1 y 4 años de estudio y vivían con familiares. La prevalencia del riesgo de caída fue del $51,7 \%$. En todas las escalas utilizadas hubo relación entre la fragilidad y el riesgo de caída $(p<0,001)$. En el análisis de regresión logística lineal, el anciano considerado frágil por la Escala de Tilburg presentó 6,05 veces más de probabilidad de caer que el no frágil. En la Escala de Groningen, la probabilidad de caída del anciano frágil fue 5,55 veces mayor. Y en la Escala de Edmonton, los que presentaron riesgo de caída obtuvieron un aumento de 1,53 en el promedio de la puntuación.

Conclusión: El riesgo de caída fue considerado de mayor significación cuando se lo relaciona con la fragilidad, en las tres escalas utilizadas. Estas escalas son instrumentos de fácil acceso y aplicación por parte de enfermeros y equipos multiprofesionales y pueden adoptarse para favorecer a un envejecimiento activo.

\section{Introdução}

O envelhecimento humano é um processo complexo, que ocasiona alteraçóes fisiológicas e sistêmicas no organismo, bem como diminuição do tônus muscular e flexibilidade e redução das terminações nervosas. Trata-se de um processo que impóe limitaçóes e gera vulnerabilidades que podem alterar as funçóes de equilíbrio dos idosos, tornando-os mais susceptíveis ao risco de queda. ${ }^{(1)}$

Segundo a Organização Mundial de Saúde (OMS), a queda é definida como um acontecimento involuntário que venha a trazer o corpo ao chão ou sobre outra superfície. ${ }^{(2)}$ Estima-se que um terço dos idosos acima de 65 anos vivencie um episódio de queda anualmente, sendo esta a segunda principal causa de morte por lesóes não intencionais no mundo. ${ }^{(3)}$ No Brasil, cerca de $30 \%$ dos idosos caem uma vez por ano, e as pessoas mais acometidas são justamente as mais idosas (80 anos ou mais de idade). ${ }^{(4)}$

A queda entre idosos é um fator de grande relevância social para a saúde pública, pois constitui uma das principais causas de lesôes, traumas, hospitalizaçóes e óbito nessa faixa etária. Além disso, esses eventos contribuem para o declínio funcional e diminuem a autonomia, com reflexos diretos na qualidade de vida do idoso. ${ }^{(5,6)}$

No processo do envelhecimento, alguns fatores intrínsecos e extrínsecos, como perda de massa muscular, diminuição de equilíbrio, comprometimento de doenças crônicas não transmissíveis, polifarmácia, queda e déficit cognitivo, comprometem a saúde e elevam o risco para a queda. Tais fatores, por sua vez, associam essa síndrome geriátrica a uma segunda: a fragilidade. ${ }^{(7)}$

O conceito de fragilidade é discutido entre pesquisadores das áreas de geriatria e gerontologia, podendo estar relacionado à idade avançada, a comorbidades e/ou incapacidades. É conceituada como "uma síndrome clínica, de natureza multifatorial, caracterizada pela diminuição das reservas de energia e pela resistência reduzida aos estressores, condiçôes essas que resultam do declínio acumulativo dos sistemas fisiológicos". ${ }^{(8)}$

Destacamos também outro conceito de fragilidade, compreendido como "uma síndrome médica com múltiplas causas e contribuintes que se caracteriza por força diminuída, resistência e função fisiológica reduzida que aumenta a vulnerabilidade de um indivíduo para desenvolver maior dependência e/ou morte". (7)

Em metanálise que reuniu 10 artigos a respeito da associação entre o risco de queda e a fragilidade, os autores verificaram, entre os 102.130 idosos com mais de 65 anos que participaram desses estudos, que 33.503 (32,80\%) deles sofreram queda. Quando comparadas ambas as variáveis, os idosos frágeis apresentavam maior risco de queda (OR 2,50; IC 95\% 1,58-3,96) quando comparados com os não frágeis e os pré-frágeis (OR 1,47; IC 95\% $1,22-1,79) .{ }^{(9)}$

$\mathrm{Na}$ análise da literatura, observamos escassez de estudos que tenham verificado o risco de queda associado simultaneamente a diferentes instrumen- 
tos de fragilidade de idosos por meio de diversos instrumentos de medidas. Assim, consideramos necessário o desenvolvimento de pesquisas que forneçam subsídios para a implementação de ações que melhorem as condiçóes de vida e a saúde dessas pessoas mais frequentemente acometidas por esses episódios. A comparação dos instrumentos de medidas pode comprovar se as quedas estão, de fato, relacionadas à fragilidade, e com esses resultados o enfermeiro e a equipe de saúde podem elaborar um plano de cuidado específico para prevenir e/ou reduzir esses incidentes nessa parcela da população. Diante do exposto, o objetivo foi analisar a associação entre o risco de queda e a síndrome da fragilidade em idosos que vivem no domicílio.

\section{Métodos}

Estudo transversal, realizado no município de Ribeirão Preto, São Paulo. Os dados foram coletados entre Novembro de 2017 e Março de 2018.

O processo de amostragem foi probabilístico, por conglomerados, de duplo estágio. No primeiro, consideramos o setor censitário como Unidade Primária de Amostragem (UPA) e sorteamos 30 setores censitários, entre os 600 existentes no município. $\mathrm{O}$ segundo consistiu em visitar um número fixo de domicílios, para garantir a autoponderação amostral. Como forma de prevenir recusas, foram sorteadas 496 pessoas, número que resulta na taxa de resposta prevista em $80 \%$, posteriormente, o número final foi de 261 participantes.

Os critérios de inclusão para participação no estudo foram: idade igual ou superior a 60 anos, ser de ambos os sexos e residir em domicílios na área urbana do município e os de exclusão foram apresentar dificuldade na comunicação.

A coleta dos dados foi realizada por alunos de graduação e pós-graduação previamente capacitados. Os instrumentos de coleta de dados utilizados foram:

- Questionário de Perfil Demográfico: permitiu caracterizar os participantes quanto a: sexo (masculino e feminino); idade (em anos completos); estado civil (solteiro, casado, separado, divorciado ou viúvo); renda do idoso (reais); escolaridade (em anos de estudos formais); número de filhos, com quantas pessoas vive o idoso e se é aposentado (sim e não).

- Mini Exame do Estado Mental (MEEM): aplicado para avaliar a função cognitiva. Encontra-se traduzido e validado para a língua portuguesa, com sensibilidade de $82,4 \%$ para analfabetos, 75,6\% para baixa e média escolaridade e $80 \%$ para alta escolaridade. Tem especificidade de 97,5\% para analfabetos, 96,6\% para baixa e média escolaridade e 95,6\% para alta. ${ }^{(10)}$ É composto de 11 questóes agrupadas em sete categorias, com o objetivo de avaliar diferentes funçóes cognitivas específicas: orientação temporal, orientação espacial, registro de três palavras, atenção e cálculo, memória da evocação das palavras, linguagem, repetir a frase, capacidade construtiva visual, seguir ordens, escrever uma frase e desenho. O escore varia de 0 a 30 pontos, porém, a escala foi revisada e houve alteraçáo nos pontos de corte: 20 para analfabetos, 25 para aqueles com 1 a 4 anos de estudo, 26,5, para pessoas com 5 a 8 anos de escolaridade, 28 para aqueles entre 9 e 11 anos e 29 para aqueles com escolaridade superior a 11 anos. $^{(11)}$

- Fall Risk Score (FRS): desenvolvida e publicada em 1993, ${ }^{(12)}$ encontra-se traduzida e validada para a língua portuguesa, ${ }^{(13)}$ com sensibilidade de $74,2 \%$, especificidade de $58,8 \%$ e acurácia de $62,5 \%$. É composta de cinco questóes agrupadas em cinco domínios: Quedas Prévias (se o idoso sofreu ou não quedas); Medicaçôes (se utiliza ou não medicamentos; se sim, qual o nome); Déficit Sensorial (qual o tipo de déficit); Estado Mental (se o idoso é ou não considerado orientado com base nos resultados do MEEM); e Marcha (qual tipo de marcha o idoso apresenta). $\mathrm{O}$ escore da escala varia de 0 a 5 pontos. Resultados finais com pontuação igual ou superior a três indicam risco de queda.

- Edmonton Frail Scale (EFS): Validada e reproduzida para a língua portuguesa. ${ }^{(14)}$ Apresentou reprodutibilidade no teste interobservador por meio do Kappa de 0,81 (IC 95\% 0,61-1,00), e 
o coeficiente de correlação interclasse alcançou 0,87 (IC 95\% 0,82-0,91, $\mathrm{p}<0,001$ ). No teste intraobservador, o Kappa de 0,83 (IC 95\% 0,72-0,94) e o coeficiente de correlação de 0,87 (IC 95\% 0,81-1,00, p<0,001) indicaram se tratar de uma escala confiável para uso população brasileira. ${ }^{(15)}$

A EFS avalia nove domínios, representados por 11 itens: Cognição; Estado geral de saúde; Independência funcional; Suporte social; Uso de medicamentos; Nutrição; Humor; Continência; e Desempenho funcional. A escala possui pontuação de 0 a 17 pontos, sendo que a maior pontuaçáo representa um nível mais elevado de fragilidade. ${ }^{(15)}$

- Indicador de Fragilidade de Tilburg (TFI): objetiva mensurar o nível de fragilidade do idoso. Esta escala encontra-se validada e reproduzida para a língua portuguesa. ${ }^{(16)}$ No que se refere à reprodutibilidade dessa escala, na avaliação de Kappa, houve concordância substancial em nove itens e concordância moderada em dois. Houve confiabilidade teste-reteste escore total TFI $r=0,88$ (IC95\%: 0,83-0,92); domínio físico $\mathrm{r}=0,88$ (IC95\%: 0,83-0,92); domínio psicológico $r=0,67$ (IC 95\%: 0,55-0,76); e domínio social $r=0,89$ (IC95\%: 0,84-0,92), para a pontuação total da escala e os escores de cada domínio. ${ }^{(16)}$

O TFI é composto de 15 questóes objetivas e autorreferidas, das quais 11 são respondidas com "sim ou não" e 4 disponibilizam também a opção "às vezes”. Essas perguntas são distribuídas em três domínios: físico, psicológico e social. A pontuação final varia de 0 a 15 pontos, sendo que escores $\geq 5$ pontos indicam que o indivíduo é frágil. ${ }^{(16)}$

- Indicador da fragilidade de Groningen (GFI): indica o nível de fragilidade do idoso e está validada e reproduzida para a língua portuguesa. ${ }^{(17)}$ É uma escala tipo Likert, com 15 itens referentes a 10 componentes: mobilidade, comorbidades, nutrição, capacidade física, cognição, visão, audição, solidão, depressão e ansiedade, pertencentes aos domínios físico, cognitivo, social e psicológico. Caracteriza o idoso como dependente ou independente, com base nas respostas "sim" ou "não" aos itens propostos. A pontuação final varia de 0 a 15 pontos, sendo que pontuaçóes $<4$ pontos indicam idosos não frágeis e $\geq 4$ pontos, idosos frágeis. ${ }^{(17)}$

$\mathrm{Na}$ análise dos dados foram calculadas, para as variáveis quantitativas, medidas de tendência central (média) e de dispersão (desvio-padrão) e, para as variáveis qualitativas, proporçóes.

$\mathrm{O}$ risco de queda em idosos foi considerado a variável dependente, classificada como sim ou não. As variáveis independentes utilizadas foram: sexo (masculino ou feminino), idade (idoso mais jovem e idoso mais velho), escolaridade (em anos), estado civil (com ou sem companheiro), mora sozinho (sim ou não) e fragilidade, mensurada pelo Indicador de Fragilidade de Groningen (frágil ou não frágil), pelo Indicador de Fragilidade de Tilburg (frágil ou não frágil) e pela Escala de Fragilidade de Edmonton (pontuação total).

Para as análises bivariadas, utilizamos o teste de Qui-quadrado de Pearson para investigar o risco de queda e a fragilidade (Escalas de Tilburg e Groningen). Já para a escala de fragilidade de Edmonton, comparamos as médias utilizando o Teste não paramétrico de Mann-Whitney.

Realizada a análise de Regressão Logística Linear com a finalidade de identificar a Razão de Chance (OR) náo ajustada entre o risco de queda com cada instrumento utilizado para verificar a fragilidade. Já para o OR ajustado foram utilizadas as variáveis sexo e idade para cada instrumento usado na avaliação da síndrome da fragilidade. Para todas as análises foi considerado $\mathrm{p}<0,05$ como nível de significância.

O projeto foi aprovado pelo Comitê de Ética em Pesquisa da Escola de Enfermagem de Ribeirão Preto da USP $n^{\circ}$ CAAE 96222418.0.0000.5393. Os participantes assinaram o Termo de Consentimento Livre e Esclarecido, em duas vias.

\section{Resultados}

Foram avaliados 261 idosos, 185 (70,9\%) do sexo feminino e 76 (29,1\%) do masculino, com idade média 
de 80,76 anos $(\mathrm{DP}=7,23)$. Em relação ao estado civil, 25 (9,6\%) eram solteiros, 103 (39,5\%) casados, 19 (7,3\%) divorciados, desquitados ou separados, 112 $(42,9 \%)$ viúvos e $2(0,8)$ outros. A média de escolaridade foi de 5,82 anos de estudo (DP $=7,62) ; 61$ $(23,4 \%)$ moravam sozinhos, 197 (75,5\%) com os familiares e $3(1,1 \%)$ com não familiares. A prevalência do risco de queda foi de $51,7 \%$. A tabela 1 apresenta as prevalências estratificadas do risco de queda segundo as variáveis sociodemográficas e as escalas de fragilidade. $\mathrm{Na}$ associação entre as variáveis demográficas e a fragilidade com o risco de queda, verificamos significância estatística com o sexo, a idade, a escolaridade e as escalas de Tilburg e Groningen (Tabela 1).

O risco de queda esteve presente em 135 (51,7\%) idosos. Quando comparamos o risco de queda com a fragilidade, $66,7 \%$ daqueles considerados frágeis pela Escala de Tilburg apresentaram este risco. Já quando aplicamos a escala de Groningen, o risco caiu para 65,2\%. Em todas as escalas, a fragilidade apareceu associada com o risco de queda $(\mathrm{p}<0,001)$ (Tabela 1$)$.

Tabela 1. Análise bivariada entre risco de queda em idosos, variáveis sociodemográficas e escalas de fragilidade $(n=261)$

\begin{tabular}{|c|c|c|c|}
\hline Variáveis & $\begin{array}{c}\text { Sem risco de } \\
\text { queda } \\
n(\%)\end{array}$ & $\begin{array}{c}\text { Com risco de } \\
\text { queda } \\
n(\%)\end{array}$ & $p$-value \\
\hline \multicolumn{4}{|l|}{ Sexo } \\
\hline Masculino & $47(37,3)$ & $29(21,5)$ & 0,005 \\
\hline Feminino & $79(62,7)$ & $106(78,5)$ & \\
\hline \multicolumn{4}{|l|}{ Idade } \\
\hline Idoso mais jovem (60-79anos) & $64(50,8)$ & $51(37,8)$ & 0,034 \\
\hline Idoso mais velho (>80anos) & $62(49,2)$ & $84(62,2)$ & \\
\hline \multicolumn{4}{|l|}{ Estado civil } \\
\hline Sem companheiro & $71(56,3)$ & $87(64,5)$ & 0,181 \\
\hline \multicolumn{3}{|l|}{ Escolaridade } & \\
\hline Analfabeto & $7(5,5)$ & $28(20,7)$ & $<0,001$ \\
\hline 1 a 4 anos & $66(52,4)$ & $76(56,3)$ & \\
\hline 5 a 9 anos & $19(15,1)$ & $15(11,1)$ & \\
\hline 10 ou mais anos & $34(27,0)$ & $16(11,9)$ & \\
\hline \multicolumn{4}{|l|}{ Mora sozinho } \\
\hline Não & $92(73,0)$ & $108(80,0)$ & 0,183 \\
\hline Sim & $34(27,0)$ & $27(20,0)$ & \\
\hline \multicolumn{4}{|l|}{ Fragilidade } \\
\hline \multicolumn{4}{|l|}{ Tilburg } \\
\hline Não frágil & $97(77,0)$ & $45(33,3)$ & $<0,001$ \\
\hline Frágil & $29(23,0)$ & $90(66,7)$ & \\
\hline \multicolumn{4}{|l|}{ Groningen } \\
\hline Não frágil & $99(78,6)$ & $47(34,8)$ & $<0,001$ \\
\hline Frágil & $27(21,4)$ & $88(65,2)$ & \\
\hline
\end{tabular}

Fragilidade de Tilburg e Groningen - Qui-quadrado

Considerando que a escala de Edmonton é numérica, sua análise foi realizada por meio da média e do desvio-padrão. $\mathrm{O}$ escore médio chegou a 6,9 pontos. Nos idosos sem risco de queda, a média foi de 3,98 ( $\mathrm{DP}=2,37)$, enquanto naqueles com risco de queda chegou a $7(\mathrm{DP}=2,59)$. Quando comparadas as médias, verificamos significância estatística $(\mathrm{p}<0,0001)$. Os modelos brutos e ajustados foram desenvolvidos para os dados das escalas de Tilburg e Groningen e, independentemente da significância estatística, mantivemos as variáveis idade e sexo. Ainda assim, testamos as variáveis estado civil (com companheiro e sem companheiro), escolaridade (analfabetos, de 1 a 4 anos de estudo, de 5 a 9 anos de estudo e de 10 anos ou a mais) e com quem mora (sozinho e acompanhado) isoladamente por escalas (Tabela 2). Na razão de chance ajustada com a Escala de Tilburg, evidenciamos que o idoso considerado frágil tem 6,05 vezes mais chances de cair do que aquele não frágil. Já na Escala de Groningen, as chances do idoso frágil cair foram 5,55 vezes maiores na comparação com o idoso não frágil. Por fim, na Escala de Edmonton (numérica), verificamos que os idosos com risco de queda tiveram um aumento de 1,53 na média no escore da escala (Tabela 2 ).

Tabela 2. Associação entre fragilidade e risco de queda segundo os modelos bruto e ajustado $(n=261)$

\begin{tabular}{lcc}
\hline Escalas de fragilidade & OR (bruto) (IC 95\%) & OR (ajustado) (IC 95\%) \\
\hline Tilburg & $6,69(3,86-11,57)$ & $6,05(3,27-11,18)^{\star}$ \\
Groningen & $6,86(3,94-11,94)$ & $5,55(3,07-10,04)^{\star \star}$ \\
Edmonton & $1,63(1,42-1,84)$ & $1,53(1,33-1,75)^{\star \star}$ \\
\hline
\end{tabular}

* 0 R ajustado para sexo, idade, com quem mora e renda do idoso; ** 0 R ajustado para sexo, idade e renda do idoso; ${ }^{* *} \mathrm{OR}$ ajustado para sexo, idade e renda do idoso

\section{Discussão}

Neste estudo, houve predomínio de idosas mais velhas, viúvas, com 1 a 4 anos de estudo e que moravam com os seus familiares. Resultados similares em relação à idade avançada e ao predomínio de idosos do sexo feminino são descritos na literatura nacional e internacional o que está relacionado com a feminização mundial da velhice. ${ }^{(18,19)}$

A idade avançada predominante é explicada por várias alteraçóes biológicas do corpo, durante o processo do envelhecimento e pelo aumento da expectativa de vida. ${ }^{(20)}$

Neste estudo, encontramos prevalência do risco de queda de $51,7 \%$, semelhante ao descrito na literatura nacional ${ }^{(21)}$ e internacional. ${ }^{(22,23)}$ 
Os motivos que levam um idoso a cair podem estar relacionados a fatores intrínsecos como o envelhecimento, patologias e uso de medicamentos. Já os extrínsecos estão relacionados ao ambiente em que ele se encontra, tais como iluminação, presença de objetos no chão, uso de roupas e calçado inadequado e dispositivos de auxílio. ${ }^{(7)}$

Em idosos, a queda pode ocasionar a perda da capacidade funcional, acarreta também lesões, fraturas ósseas, hospitalizaçóes e mortes, o que torna a alta prevalência desses eventos nesta parcela da população um problema de saúde pública. ${ }^{(24)}$

Estudos apontam que o risco de queda em idosos pode estar associado ao sexo feminino, devido à fisiologia humana, uma vez que a estrutura óssea e muscular das mulheres é mais frágil e elas vivenciam alterações hormonais específicas deste sexo, além de apresentarem maior número de doenças crônicas e estarem mais expostas a tarefas domésticas. ${ }^{(13,25)}$ Neste estudo, no entanto, observamos a presença de outro fator preditor para o risco queda e que merece atenção: a síndrome da fragilidade.

As ocorrências de quedas no idoso estão diretamente relacionadas com a idade avançada e o grau de fragilidade (OMS). ${ }^{(26)}$ Estudo de metanálise que avalia a associação entre o risco de queda e a fragilidade em 102.130 idosos com mais de 65 anos, mostra que idosos frágeis apresentam maior risco de queda comparado aos não frágeis. ${ }^{(9)}$

Em pesquisa realizada no Arizona com 119 idosos, 48 deles relataram um ou mais episódios de quedas ao longo da vida, sendo que $47 \%$ eram idosos frágeis. $\mathrm{O}$ déficit de equilíbrio e o hábito de praticar caminhadas foram os preditores mais sensíveis para queda associados a idosos pré-frágeis e frágeis. ${ }^{(27)}$

$\mathrm{Na}$ análise de regressão logística, evidenciamos que a chance de um idoso frágil sofrer queda é maior comparado ao idoso não frágil, independentemente das escalas serem categóricas ou numéricas e das variáveis demográficas investigadas. Observamos que, quando aplicadas as escalas de Tilburg e Groningen, que são categóricas, os idosos classificados como frágeis apresentaram maior risco de queda do que aqueles classificados como não frágeis. Já mediante a aplicação da escala de Edmonton, que é numérica, o idoso frágil apresentou, a cada ponto do escore, mais chances de sofrer um episódio de queda do que o idoso não frágil. As medidas de todas as escalas utilizadas foram significantes para estimar o risco de queda entre os participantes.

A relação entre queda e condição de fragilidade de idosos que vivem na comunidade chinesa foi investigada em estudo, cujos resultados mostraram que quanto maior o índice de fragilidade, maior a chance de uma pessoa idosa apresentar este risco, em comparação com outros fatores como sexo e idade. ${ }^{(28)}$ Já estudo realizado na Espanha envolveu 183 idosos acima de 69 anos de idade e avaliou o risco de queda em relação ao fenótipo da fragilidade. Constatou-se, por meio da Falls Efficacy Scale International (FES-I), que os idosos frágeis estavam mais suscetíveis a novos episódios de queda se comparados aos não frágeis $\left(\mathrm{OR}=3,18\right.$; IC95\% 1,32-7,65). ${ }^{(19)}$ Eles também se mostraram mais preocupados com a possibilidade de sofrerem uma nova queda (OR=3,93; IC95\% 1,85$8,36)$, com valores finais ajustados associados ao sexo feminino e ao risco de depressão. ${ }^{(19)}$

A fragilidade diminui a resistência física, altera o equilíbrio, potencializa o quadro de fraqueza e reduz o desempenho motor do idoso, causando um declínio da reserva fisiológica do organismo e fazendo com que ele fique vulnerável a eventos adversos, inclusive a quedas. ${ }^{(8)}$ Embora sejam síndromes distintas, a fragilidade está associada à queda em virtude da perda de massa muscular, que pode ocasionar sarcopenia, do desenvolvimento de doenças crônicas, do uso de medicamentos, do déficit cognitivo e de episódios de delírio, fatores que notoriamente aumentam o risco de um idoso cair. ${ }^{(7)}$

Como limitaçóes deste estudo, destacamos a ausência de pesquisas que tenham utilizado mais de uma escala para estimar o risco de queda associado à fragilidade, o que dificultou a discussão dos dados e o debate de ideias. Uma segunda limitação é o tipo do estudo, transversal, que não nos permite inferir se a fragilidade leva o idoso a sofrer a queda ou vice-versa, sendo necessário realizar um seguimento com esses idosos para esclarecer essa dúvida. A terceira envolve a possibilidade de que a queda seja influenciada por fatores extrínsecos, por exemplo, iluminação, superfície do piso e presença de tapetes e/ou degraus, contexto que não foram investigados, embora elevem o risco de queda. 


\section{Conclusão}

O evento queda em idosos é um problema de saúde pública, especialmente quando envolve aqueles frágeis. Verificamos que com uso de diferentes escalas para avaliar a fragilidade é possível identificar o risco de queda, sendo que com a Escala de Groningen este risco é maior. As três escalas utilizadas no presente estudo apresentam características distintas: as de Groningen e Tilburg são categóricas e a de Edmonton, numérica. No entanto, as escalas de per si conseguem predizer o risco de queda em idosos que moram no domicílio e foram unânimes em associar este risco à fragilidade. Sáo instrumentos de fácil aplicação por parte do enfermeiro e equipe multiprofissional, capazes de identificar e prevenir o risco de queda, o que pode favorecer um envelhecimento mais ativo. Dessa forma, este estudo, ao analisar a associação entre o risco de queda e a síndrome da fragilidade em idosos que vivem no domicílio, mediante a aplicação das três escalas, mostrase fundamental, na medida em que os profissionais de saúde podem ter acesso a esses instrumentos e utilizá-los para prevenir quedas em idosos.

\section{Agradecimentos}

Este estudo foi financiado pelo Conselho Nacional de Desenvolvimento Científico e TecnológicoCNPq, processo número 305565/2016-8.

\section{Colaborações}

Giacomini SBL, Fhon JR e Rodrigues RAP declaram que contribuíram com a concepção do projeto, análise e interpretação dos dados, revisão crítica relevante do conteúdo intelectual e aprovação da versão final a ser publicada.

\section{Referências}

1. Landinez PN, Contreras VK, Castro VA. Proceso de envejecimiento, ejercicio y fisioterapia. Rev Cuba Salud Pública. 2012;38(4):54280.
2. Organización Mundial de la Salud (OMS). Caídas: nota descriptiva [Internet]. Geneva: OMS; 2016. [citado 2019 Jan19]. Disponível em: http://www.who.int/mediacentre/factsheets/fs344/es/

3. Organização Mundial de Saúde [OMS]. Quedas [Internet]. Geneva: OMS; 2018 [citado 2019 Jan 19]. Disponível em: http://www.who.int/ news-room/fact-sheets/detail/falls.

4. Ansai JH, Glisoli SF, Oliveira T, Soares AT, Cabral KN, Sera CT, et al. Revisão de dois instrumentos clínicos de avaliação para predizer risco de quedas em idosos. Rev. Bras. Geriatr. Gerontol. Riso. 2014;17(1):177-89.

5. Tinetti ME, Kumar C. The patient who falls: "It's always a trade-off". JAMA. 2010;303(3):258-66.

6. Moraes AS, Soares WJ, Lustosa LP, Bilton TL, Ferrioli E, Perracini MR. Características das quedas em idosos que vivem na comunidade: estudo de base populacional. Rev Bras Geriatr Gerontol (Rio DE Janeiro). 2017;20(5):693-704.

7. Morley JE, Vellas B, van Kan GA, Anker SD, Bauer JM, Bernabei R, et al. Frailty consensus: a call to action. J Am Med Dir Assoc. 2013;14(6):392-7.

8. Fried LP, Tangen CM, Walston J, Newman AB, Hirsch C, Gottdiener J, et al.; Cardiovascular Health Study Collaborative Research Group. Frailty in older adults: evidence for a phenotype. J Gerontol A Biol Sci Med Sci. 2001;56(3):M146-56.

9. Cheng MH, Chang SF. Frailty as a risk factor for falls among community dwelling people: evidence from meta-analysis. J Nurs Scholarsh. 2017;49(5):529-36.

10. Bertolucci PH, Brucki SM, Campacci SR, Juliano YO. Mini-Exame do Estado Mental em uma população geral. Arq Neuropsiquiatr. 1994;52(1):1-7.

11. Brucki SM, Nitrini $R$, Caramelli P, Bertolucci PH, Okamoto $\mathbb{H}$. Sugestões para 0 uso do mini-exame do estado mental no Brasil. Arq Neuropsiquiatr. 2003;61 3B:777-81.

12. Downton JH. Falls um the elderly. London: British Library Cataloguing; 1993. $158 \mathrm{p}$

13. Schiaveto FV. Avaliação do risco de quedas em idosos na comunidade [dissertação]. Ribeirão Preto (SP):Universidade de São Paulo, Escola de Enfermagem de Ribeirão Preto ; 2008.

14. Fabricio-Wehbe SC, Schiaveto FV, Vendrusculo TR, Haas VJ, Dantas RA, Rodrigues RA. Cross-cultural adaptation and validity of the Edmonton Frail Scale - EFS in a Brazilian elderly simple. Rev Lat Am Enfermagem. 2009;17(6):1043-9.

15. Fabricio-Wehbe SC, Cruz IR, Haas VJ, Diniz MA, Dantas RAS, Rodrigues RAP Reproducibility of the Brazilian version of the Edmonton Frail Scale for elderly living in the community. Rev Lat Am Enfermagem. 2013;21(6):1330-6.

16. Santiago LM, Luz LL, Mattos IE, Gobbens RJ. Adaptação transcultural do instrumento Tilburg Frailty Indicator (TFI) para a população Brasileira. Cad Saude Publica. 2012;28(9):1795-801.

17. Borges CL. Adaptação transcultural do instrumento Groningen Frailty Indicator em idosos brasileiros [dissertação]. Fortaleza (CE): Universidade Estadual do Ceará, Centro de Ciências da Saúde; 2013.

18. Smith AA, Silva A0, Rodrigues RA, Moreira MA, Nogueira JA, Tura LF. Assessment of risk of falls in elderly living at home. Rev Lat Am Enfermagem. 2017;25(0):e2754.

19. Esbrí-Víctor M, Huedo-Rodenas I, López-Utiel M, Navarro-López JL, Martínez-Reig M, Serra-Rexach JA, et al. Frailty and fear of falling: the fistac study. J Frailty Aging. 2017;6(3):136-40.

20. Freitas MG, Bonolo PF, Moraes EN, Machado CJ. Idosos atendidos em serviços de urgência no Brasil: um estudo para vítimas de quedas e de acidentes de trânsito. Cien Saude Colet. 2015;20(3):701-12. 
21. Vieira LS, Gomes AP, Bierhals I0, Farías-Antúnez S, Ribeiro CG, Miranda VI, et al. Quedas em idosos no Sul do Brasil: prevalência e determinantes. Rev Saude Publica. 2018;52:22.

22. Kistler BM, Khubchandani J, Jakubowicz G, Wilund K, Sosnoff J. Falls and fall-related injuries among us adults aged 65 or older with chronic kidney disease. Prev Chronic Dis. 2018;15:E82.

23. Ouyang $P$, Sun $W$. The association between depressive symptoms and fall accidents among middle-aged and elderly people in China. Environ Health Prev Med. 2018;23(1):42.

24. Steinman BA, Pynoos J, Nguyen AQ. Fall risk in older adults: roles of self-rated vision, home modifications, and limb function. J Aging Health. 2009;21(5):655-76.
25. Mohler MJ, Wendel CS, Taylor-Piliae RE, Toosizadeh N, Najafi B. Motor performance and physical activity as predictors of prospective falls in community-dwelling older adults by frailty level: application of Wearable Technology. Gerontology. 2016;62(6):654-64.

26. World Health Organization (WHO). Good health adds life to years: Global brief for World Health Day 2012. Geneva: Who; 2012.

27. Tom SE, Adachi JD, Anderson FA Jr, Boonen S, Chapurlat RD, Compston JE, et al.; GLOW Investigators. Frailty and fracture, disability, and falls: a multiple country study from the global longitudinal study of osteoporosis in women. J Am Geriatr Soc. 2013;61(3):327-34.

28. Zhou BY, Yu DN, Tao YK, Shi J, Yu PL. [Relationship between fall and frailty index in elderly adults of urban community in Beijing]. Zhonghua Liu Xing Bing Xue Za Zhi. 2018;39(3):308-12. Chinese. 